\title{
System Requirements Model for Multi-Agent Autonomic Fetus Monitoring System
}

\author{
Rasika Mallya ${ }^{1,2 *}$ and Snehalata Kothari ${ }^{1}$ \\ 'PAHER University, Pacific Hills, Pratapnagar Extn, Airport Road, Debari, Udaipur - 313003, \\ Rajasthan, India; skothariudr@gmail.com \\ ${ }^{2}$ Navinchandra Mehta Institute of Technology and Development, Dess Mumbai Campus, Kirti College Road, \\ Dadar West, Mumbai - 400028, Maharashtra, India; rasikamallya@gmail.com
}

\begin{abstract}
In this paper, a new fetal monitoring system is presented using wearable technology. This wearable devise is equipped with automatic multi-agent software to monitor fetus's and mother's body parameter from the first trimester of conception. The system generates alerts when body parameters of a mother and fetus change abnormally. If the parameters are changed drastically, the system sends the alert message to obstetrician of gravida and also to family caretaker. This system uses PASSI technology. This approach helps to keep watch on fetus development remotely since monitoring data will be stored by the system. It reduces hospital trips and saves time and efforts of hospital staff for monitoring gravida frequently. PASSI technology can create system requirements models which identifies agents and creates task specification of agents.
\end{abstract}

Keywords: Autonomic Fetus Monitoring System, Multi-agent System, PASSI Technology, System Requirements Models, Wearable Technology

\section{Introduction}

Due to diversity, heterogeneity and advances in technology, fetal monitoring, has made remarkable progress. Over the past few decades, electronic fetal monitoring systems have emerged as a very promising tool for use by midwives, obstetricians, and labor and delivery nursing staff. Further, wearable devices have provided unbiased, accurate data for monitoring patient's activity and this has enabled the healthcare community to monitor patient's outcomes and analyze overall trends. Su and $\mathrm{Chu}^{1}$ have presented a mobile system for fetal monitoring, however, it does not monitor the fetus from the first trimester. This paper presents a wearable device equipped with autonomic multi-agent software to monitor fetus's and mother's body parameters when mother is busy in her work, right from the first trimester of conception. This devise will help the working mothers to monitor fetus, It will monitor all the activity till the device in ON. The device will monitor water level, fluid level, fetus's movements and heart beats. Traditionally, doctors and nurses could only periodically and physically monitor the baby's heartbeats using sonography or colour Doppler methods. This approach did not allow them to detect changes in fetal heartbeats at any time or offer continuous surveillance. Thus, physicians still lacked the important information for reducing perinatal or neonatal mortality rates. The proposed device will help physician to monitor fetal safety. When the water level goes down in mother's body, or the baby does not show any movement for long interval of time, the device will generate alert message for mother. So she will be alert and may drink water to maintain appropriate water level. If mother's water level or fluid level become very low or BP level becomes abnormal then monitoring system will detect and record the changes and send alert message to home caretaker and obstetrician. So they can reach patient immediately or suggest medicine on call. If the emergency index is very high then the monitoring system can also track the nearest ambulance to send patient to hospital.

The monitoring data will be stored as patterns. The collected data patterns will be useful to determine the

*Author for correspondence 
status of fetus during prenatal stage or birth process. Such fetal remote monitoring system will allow pregnant woman to track fetus development as well as it will reduce the number of hospital trips. If some abnormal patterns of data are detected, then the obstetrician can suggest medicines to pregnant woman on call. If the abnormality is severe, then the obstetrician may tell her to visit the hospital. The proposed system is useful in the scenario when the pregnant woman is engrossed in her work and forgets to drink water or take food or forgets to keep a watch on baby movements, especially in second and third trimester. The agent at front end is wearable technology which will monitor and generate patterns. These patterns will be stored in storage. At the backend there will be mobile app which will be connected to patient's remote mobile application.

\section{Research Objective}

1. The objective of this study is to perform analysis of multi-agent autonomic fetus monitoring system and identify agents and interactions among the agents for the system.

2. The major contribution of this study will be to propose autonomic system which allows immediate communication and exchange of real time patterns with gynecologist or caretaker.

3. Thus fetus and pregnant woman's safety is improved and medical staff is relieved from tedious data monitoring task and during repeated hospital visits.

\section{Literature Review}

\subsection{Agent Oriented Software Engineering}

Agent oriented software engineering applies software engineering and artificial intelligence principles to analysis, design and implementation of software systems. Agent-oriented methodologies mainly cover analysis and design of software development. Agent oriented software engineering is based on agent-oriented approach ${ }^{2}$.

- Agent-oriented approach is suitable for systems where complex communication is required.

- This approach is suitable for the system whose some of the situations are unidentified.

- In agent-based approach, agents cooperate with each other autonomously.
- The agent-based approach is useful for software development where requirements can be modified or expanded and so the system.

Using agent oriented software engineering, multi-agent system architecture is defined as: a unique entity, environment $E$, set of objects $\mathrm{O}$ in $\mathrm{E}$, set of active objects i.e. agents $A$, set of relations $\mathrm{R}$ which define relationships among the agent, set of operations, $\mathrm{O}$, which agents will perform on objects, the laws to control operations (ontology). In this paper, for the analysis and design of multi-agent autonomic fetus monitoring system, agent-oriented approach is applied 3 .

\subsection{PASSI}

It is a Process for Agents Societies Specification and Implementation. It is a step-by-step requirement-to-code methodology for designing and developing multi-agent system. The development of software using PASSI methodology is iterative and incremental as shown in Figure 1.

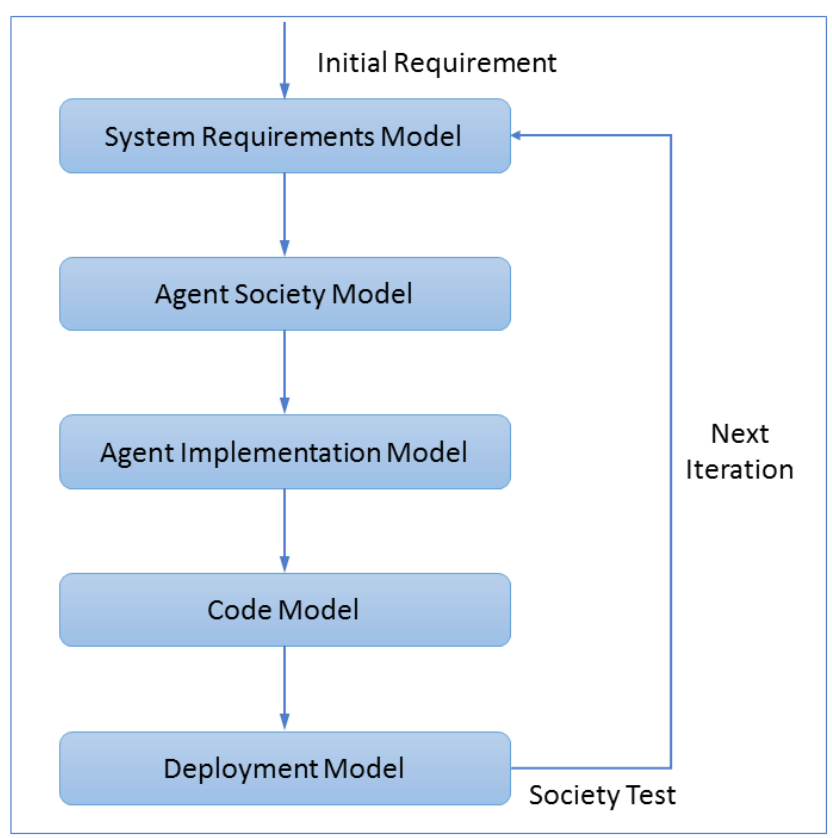

Figure 1. PASSI process flow.

During systems requirements modeling, the agent is autonomous entity which can take decisions, implement actions and has social relationships with other agents in organization. The agent performs sequence of tasks as a role. When agent implements some behavior in collaboration with itself or with other agents, it will be considered as a task. Each agent's behavior is mentioned in ontology. 


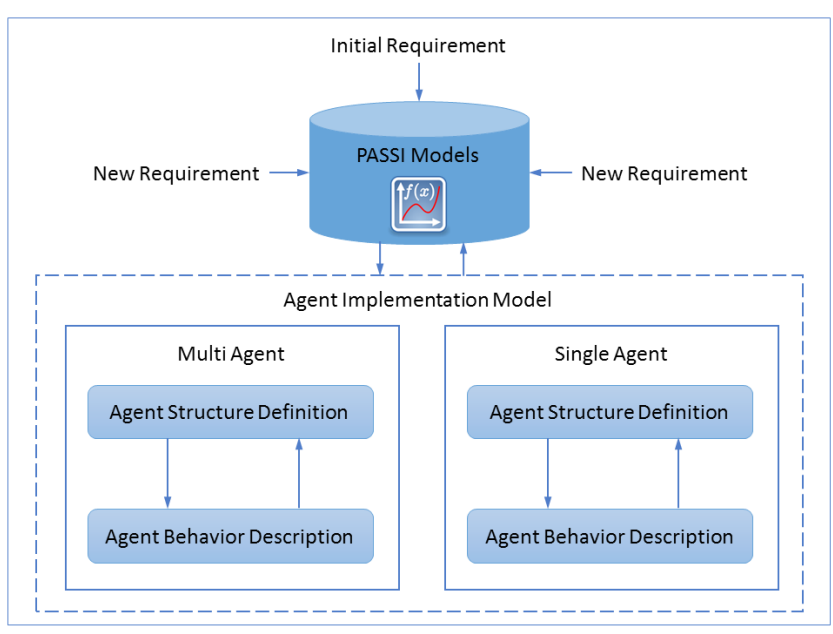

Figure 2. Iterative and incremental nature of PASSI for self-adaptivity.

The iterative nature of PASSI supports self-adaptation of requirements for agents. First iteration is for new requirements and all PASSI models are implemented with set of requirements. The second iteration creates modification in agent implementation models. During agent implementation, the implementations are done for single agent as well as for multi-agents. The multi-agents implementation models modify cooperation, task and behavior among multiple agents. The single agent implementation models modify attributes, methods and inner classes. During both implementations, agent structures and behaviors are updated. Thus, agent behavior and structures are changed as requirements are changed. The self-adaptivity in multiagents using PASSI methodology is as shown in Figure 24.

\subsection{Existing Systems for Fetal Monitoring}

Hammar and Hewlett Packard developed first commercial electronic fetal monitoring device in 1978 ${ }^{1}$. This device was used to monitor heart cycle, heart sound during labor. Jansuz developed fetal heart rate signal processing. Czabanski, R.; Jezewski, M.; Wrobel, J.; Jezewski, J.; Horoba, K. developed artificial neural network to evaluate risk of low fetal birth weight using cardiography signals 5 . New innovations are going on for electronic fetal monitoring using wireless communication technologies, portable monitoring devices like RFID. Fetal data can be transmitted to remote locations via wireless communication devices.

A research group of Sandy Pentland (Chuan-Jun Su and Ta-Wei Chu) identified location, movement and communication data from smart phone to identify flu and gastrointestinal problems. William Kaiser from UCLA developed healthcare software for stroke patient monitoring and guidance for rehabilitation over 20 countries $^{6}$.

Location Tracking Services: With the help of GPS, location estimation mechanism, location tracking services can be developed for elderly, visually impaired and intellectually disabled patients

Sleep Analysis: The patient sleeping patterns can be monitored using gyroscope and magnetometer. These devices are embedded into smart phones. The sleeping behavior is monitored by strapping the mobile phone on arm or on waist or keeping mobile below the pillow ${ }^{6}$.

Vital Sign Monitoring: There are iPhone add-on modules and mobile apps to record heart rate, ECG, blood pressure and blood sugar using Personal Area Network. This data is stored as patterns and can be useful for physician when needed 1 .

\section{System Requirements Models for MAA-FMS}

When the pregnant woman is busy in her work (office work or house work), she may neglect her water, food intakes or for intake of food and water when necessary. If heart rate, BP or uterine contractions are found abnormal, the baby movements. The illiterate pregnant woman may not know the quantity of water, food she has to take to grow fetus properly. If water intake is not in adequate quantity, the fetus may dehydrate. Inadequate food intake can cause malnutrition of fetus. It is practically impossible for obstetrician to keep watch on daily intake of water and food of mother as well as baby movements for $24^{*}$ 7. Hence, it is essential to develop a system which works with wearable device and generates alerts device will generate alert for caretaker and obstetrician of the patient.

To satisfy these requirements, this research proposes Multi-Agent Autonomic - Fetus Monitoring System (MAA-FMS) in which wearable device will collect monitoring data and spontaneously inform pregnant woman for intake and caretakers about vital signs. The MAA-FMS consists of two parts: first component is front end MAAFMS which will be the wearable device which pregnant woman will wear. Second component is back-end MAAFMS which will be with caretaker agent and obstetrician agent. The functional view of the system is as shown in Figure 3. 


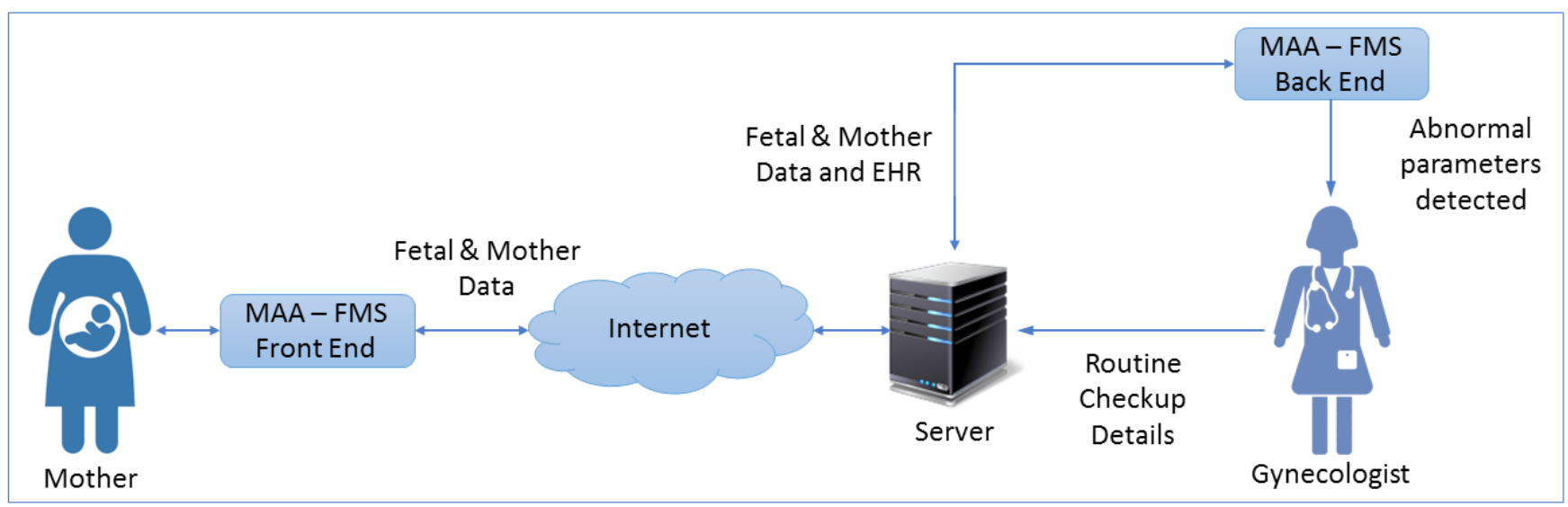

Figure 3. Functional view of MAA-FMS.

The system's agents behavior will change autonomously according to scenario at runtime, so the interactions among multi-agents will also change. The change in runtime interactions is supported by individual agent's internal structure. Here PASSI (Process for Agents Societies Specification and Implementation) methodology is suitable for requirements engineering of mentioned system. According to PASSI process flow, during requirements engineering activities executed are shown in Figure $4^{z}$.

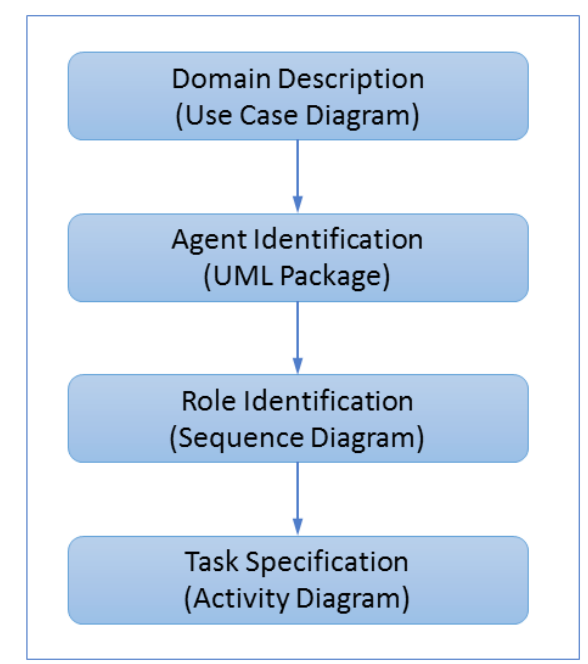

Figure 4. Requirements engineering models using PASSI.

\subsection{Domain Requirements Description Phase}

This phase describes functional description of the system in hierarchical series of use case diagrams. Two perspectives can be considered to describe domain requirements for MAA-FMS. One perspective is accord- ing to gravida where she will make the wearable device "ON" before sitting on work desk. The device will start monitoring food and water intake for gravida and fetus. If the diagnostic agent senses some abnormal parameters, it will inform wearable device and generate alerts for gravida and caretakers. All monitored data will be stored at knowledge -based data server so that whenever obstetrician needs data, it can be downloaded. The domain description model for gravida's perspective is as shown in Figure 5.

Second perspective is where obstetrician can retrieve the recorded data for specific gravida when an alert is received for her from MAA-FMS. Obstetrician can immediately monitor the recorded data patterns and suggest medicine to gravida on phone. If emergency situation is observed, he can advise gravida to visit hospital for emergency treatment. The domain description model for gynecologist's perspective is as shown in Figure 6.

\subsection{Agents Identification Phase}

Agents identification is done from domain description models. One or many use cases are grouped and converted into stereotyped packages. Each package defines functionality of each agent. As shown in Figure 7, the agents identified are GravidaUI, WearableDeviceUI, DiagnosticAgentUI and MedicalAgent. GravidaUI and WearableDeviceUI agents works for gravida, WearableDeviceUI is also responsible to store monitored data at knowledge base data server. The agents DiagnosticAgentUI and MedicalAgent work for obstetrician and caretaker. 


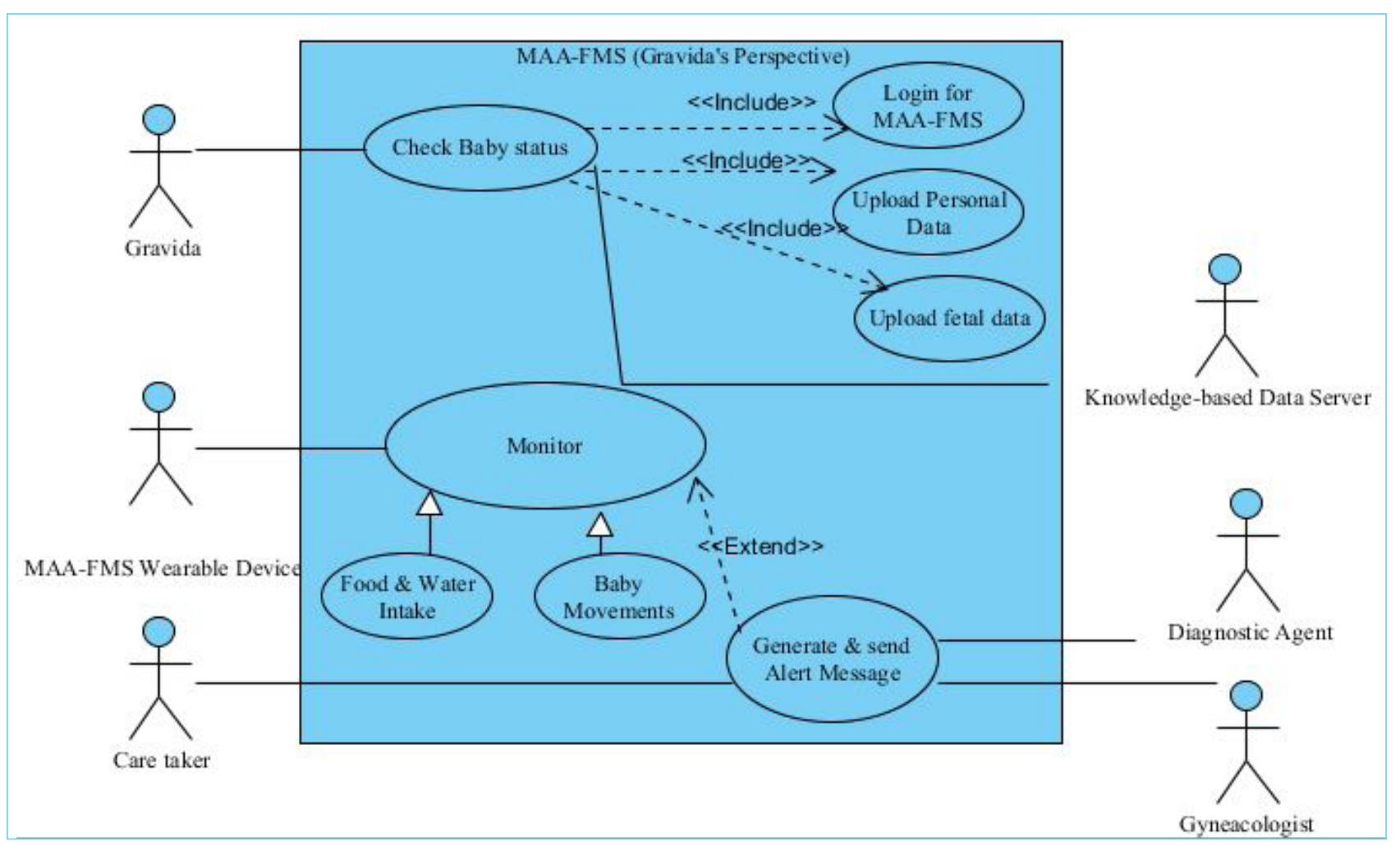

Figure 5. Domain requirements model for Gravida.

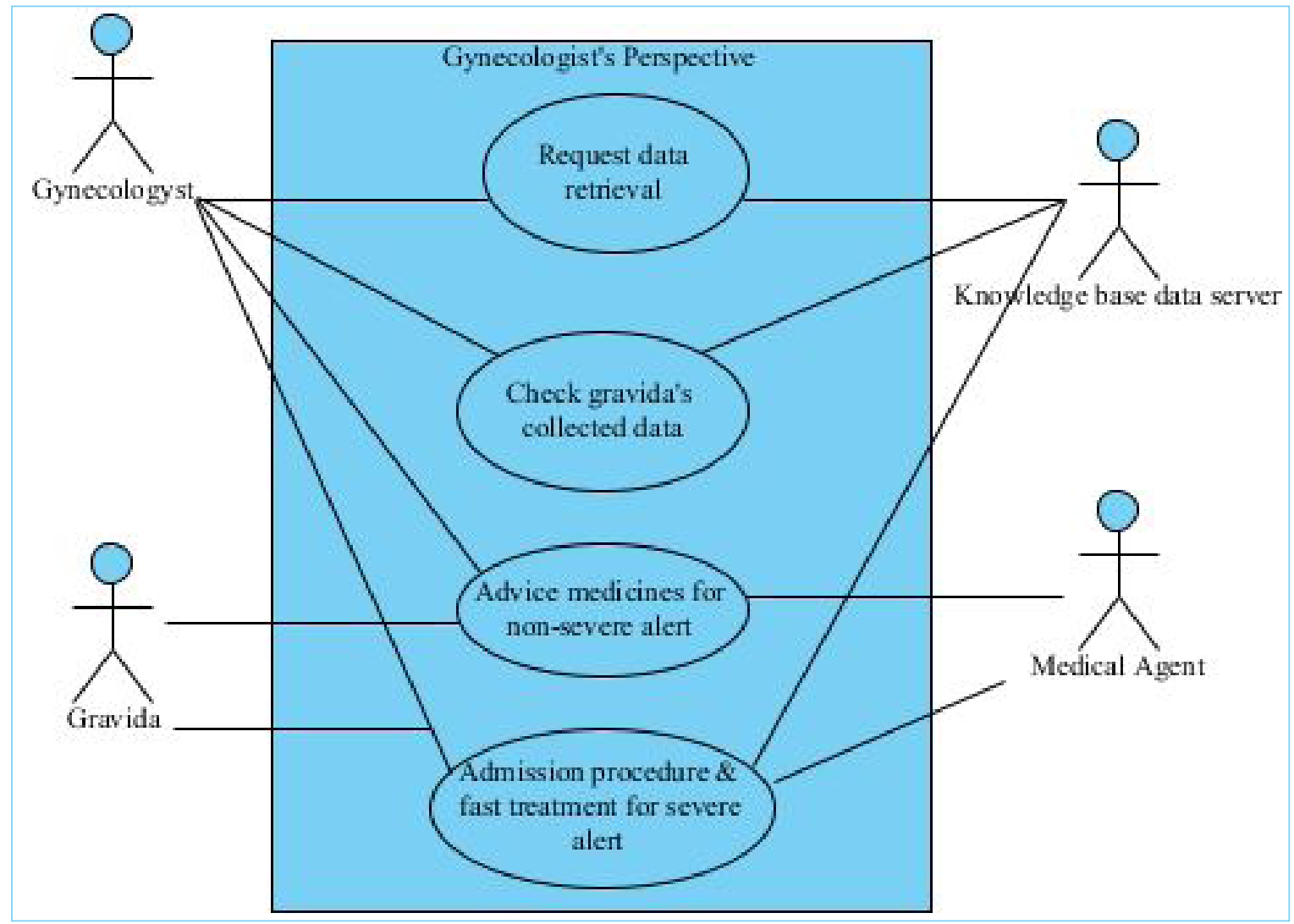

Figure 6. Domain requirements model for gynecologist. 


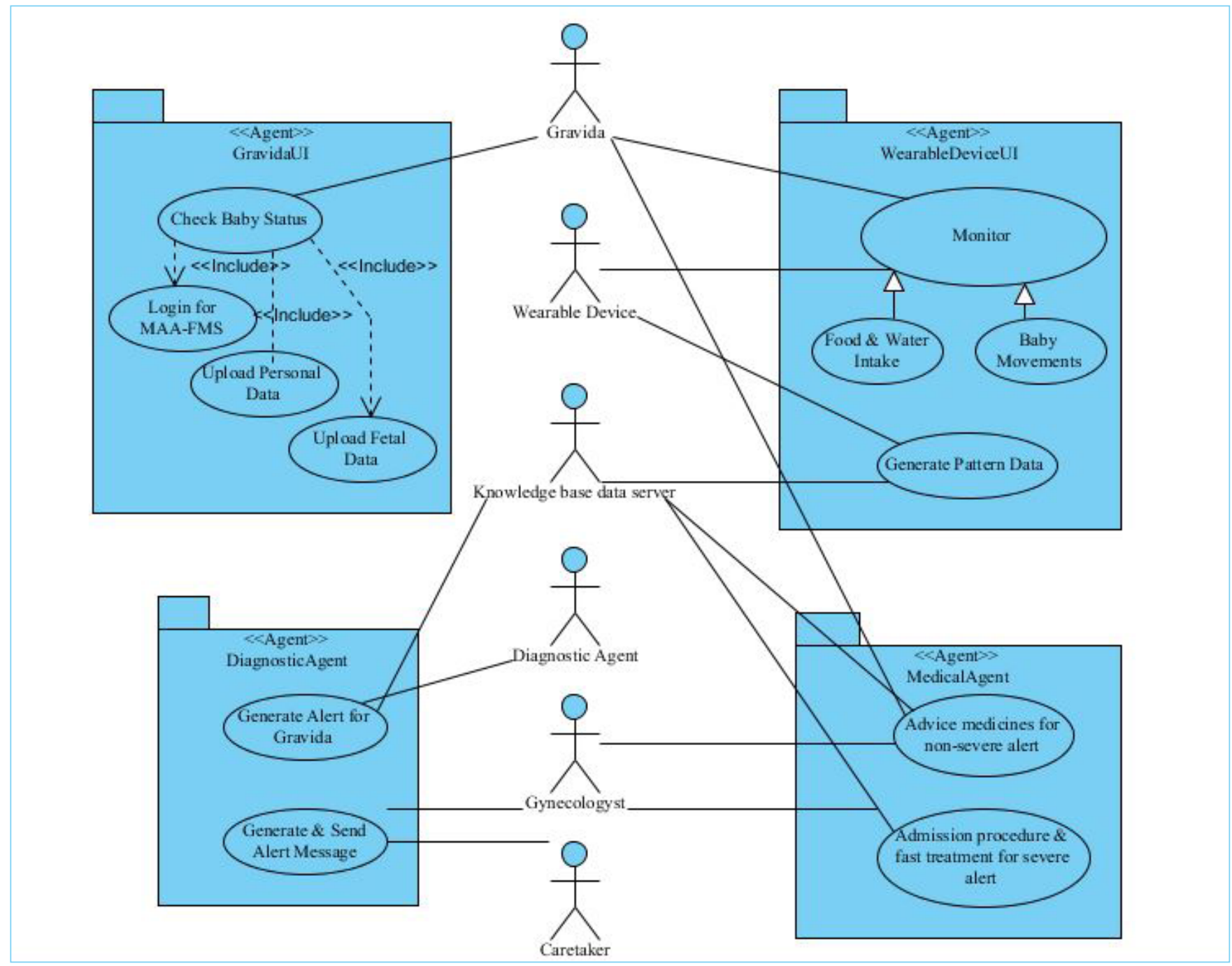

Figure 7. Agents identification model for MAA-FMS.

As shown in agent's identification model, all autonomous agents form the organization where agents perform their roles in coordination. If agents' goals are not achieved, then according to PASSI methodology the agents and their tasks are reorganized to achieve goals effectively.

\subsection{Role Identification Phase}

Roles identification is based on evaluating all possible interactions among agent which is also called inter-agent communication. Multiple scenarios are identified based on requirements and goals of agents. Each scenario is described with interacting agents and multiple communication path among them. Role identification of agent is represented using sequence diagram in $\mathrm{PASSI}^{\mathrm{Z}}$. An agent may participate in different scenarios with different roles. Each object is represented as

$$
<\text { role-name }>\text { :<agent-name }>
$$

Consider the routine scenario where gravida is working in her bank, doing some transactions. She is in her second trimester and has probably she has completed 15 or 16 weeks of pregnancy. She had her breakfast in the morning and is sitting on her work desk for about 90 minutes. The MAA-FMS wearable device is ON, keeping watch on baby movements. After 90 minutes the device detects that the water level for gravida and fetus is not adequate, it raises alert for gravida, she realizes she has not taken water from last 90-100 minutes and immediately drinks water. This scenario is shown in Figure 8 to describe role of gravida, MAA-FMS wearable device. 


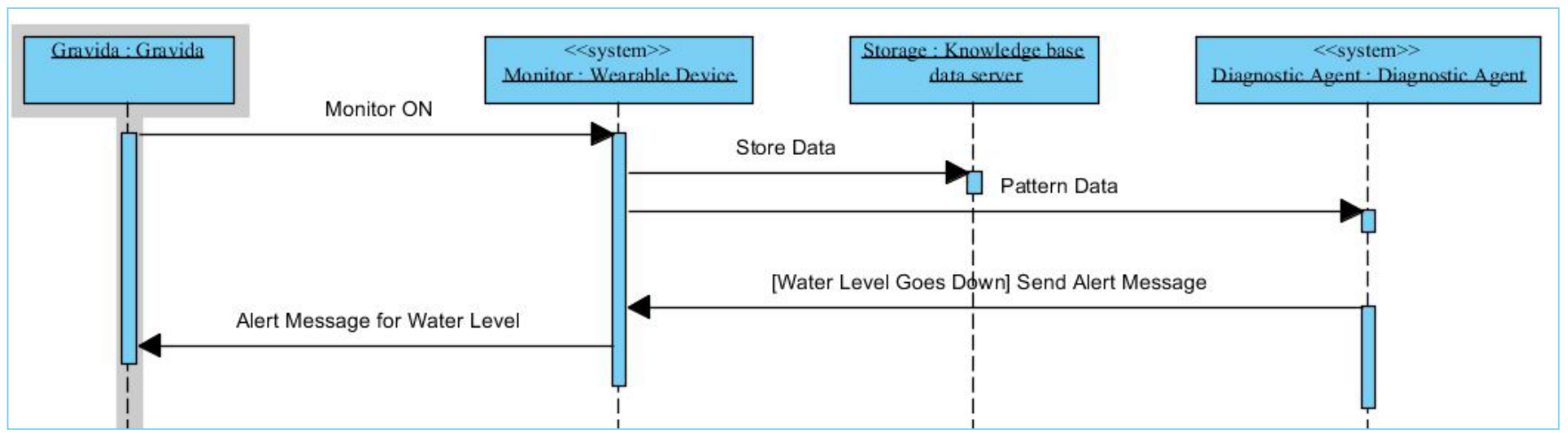

Figure 8. Role identification model for scenario 1.

Consider scenario 2 where gravida is under stress because something goes wrong while handling cash on work desk in bank. Gravida is not able to tally the cash for 2 hours. After sometime gravida's BP shoots up and she starts feeling dizziness and uncomfortable. She has faced such technical problem earlier, hence ignores the dizziness and focuses on resolving the issue. She misses to relate such stress with her current physical situation. The MAA-FMS wearable agent detects high BP and immediately generates and sends alert message for caretaker as well as obstetrician. The obstetrician's assistant calls gravida and suggests the medicine. Gravida's husband then reaches her office with medicine and help normalize the BP. This scenario is shown in Figure 9 to describe role of MAA-FMS wearable device, diagnostic agent and caretakers.

\subsection{Task Specification Phase}

Task is the collection of some actions which forms logical unit of work. For task specification model, activity diagram is drawn specific to agent. The task specifica- tion model represents agents' capabilities. Relationships among activities signify messages among activities or interactions among tasks or agents. Consider the task specification model for wearable agent for MAA-FMS. It is shown in Figure 10.

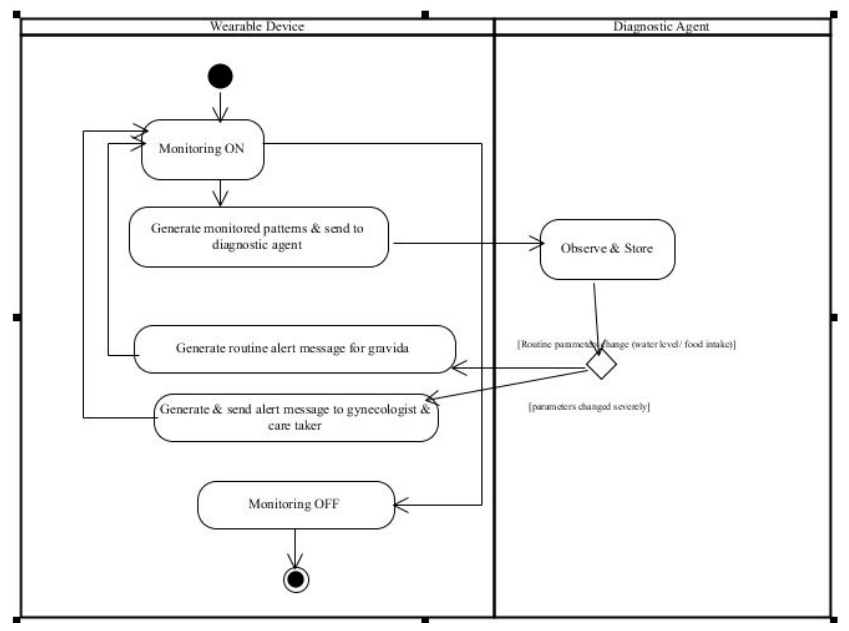

Figure 10. Task specification model.

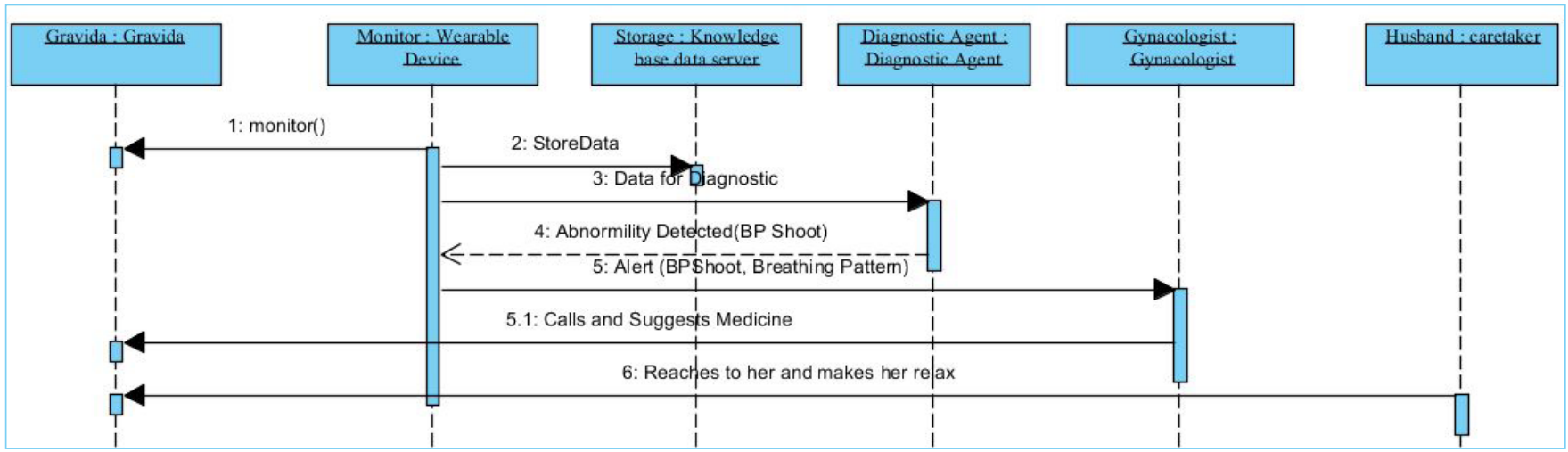

Figure 9. Role Identification model for scenario 2. 


\section{Design Phase- implementation of Requirements Model}

The domain requirements models are implemented for agent society modeling phase i.e. designing phase using PASSI for multi-agent autonomic software. Agent society models are created with the help of domain ontology description. The ontologies represent knowledge about agents and scenarios of the system. The ontology is used to support the interaction among the agents i.e. at runtime through reasoning. Roles description phase is used to represent dependencies among agents which identifies communication capabilities of agents. Further, task specification phase is tested and implemented for protocols description of system where agent interaction protocols are formed using FIPA. This is succeeded by Agents Structure Definition Phase where agent behavior description is generated for single agent as well as multi-agent structure. The current requirements models are implemented in CASE tool Visual Paradigm. The requirements models and ontologies for agent society will be implemented in PASSI toolkit, hence iterations according to change in requirements can be incorporated easily.

\section{Conclusion}

The purpose of this study is to propose Multi-Agent Autonomic- Fetus Monitoring System to monitor fetus movements, water and food intake of pregnant woman when she is engrossed in her work. The body parameters are monitored and stored for further reference of gynecologist. Here requirements models are proposed for analysis of the system. The next step of the study is to design ontology for agents and roles of agents for the system. Incorporating sophisticated diagnostic methodologies into MAA-FMS can effectively enhance the functionality of wearable device and diagnostic agent.

\section{References}

1. Su C-J, Chu T-W. A mobile multi-agent information system for ubiquitous fetal monitoring. International Journal of Environmental Research and Public Health. 2014; 11(1):600-25. Crossref.

2. Sturm A, Shehory O. Agent-oriented software engineering: Revisiting the state of the art. Springer-Verlag Berlin Heidelberg; 2014.

3. Genza NR, Mighele ES. Review on multi-agent oriented software engineering implementation. International Journal of Computer and Information Technology. 2013 May; 2(3).

4. Cossentino M. From requirements to Code with the PASSI methodology. Agent-Oriented Methodologies; 2012 Apr. Crossref.

5. Su C-J, Shih SC. Building distributed E-healthcare for elderly using RFID and multi-agent. International Journal of Engineering Business Management. 2011;3(1):16-26. Crossref.

6. Ng JK-Y. Ubiquitous healthcare: Healthcare systems and applications enabled by mobile and wireless technologies. June 2012, Volume 3 , Number 2, Journal of Convergence.

7. Cossentino M, Pott C. A CASE tool supported methodology for the design of multi-agent systems [Internet]. Available from: https://www.researchgate.net/ publication/2524911_A_CASE_tool_supported_methodology_for_the_design_of_multi-agent_systems.

8. Odell J, Parunak HV, Bauer B. Extending UML for Agents. Practical Foundations of Business System Specifications; 2003. p. 245-70.

9. Wooldridge M, Jennings NR, Kinny D. A methodology for agent-oriented analysis and design [Internet]. Available from: http://www.cs.ox.ac.uk/people/michael.wooldridge/ pubs/agents99.pdf

10. Hamilton K. Examining the multiagent systems engineering methodology. SENG 609.22. Tutorial Report.

11. Czabanski R, Jezewski M, Wrobel J, Jezewski J, Horoba K. Predicting the risk of low-fetal birth weight from cardiotocographic signals using ANBLIR system with deterministic annealing and bm $\varepsilon$-insensitive learning. IEEE Transactions on Information Technology in Biomedicine. 2010; 14:106274. Crossref. PMid:20129872 